\title{
Things that go bang in the night
}

\author{
With a growing coverage of the night sky, the quantity and quality of transient event detections is booming. In this \\ issue, our Focus looks particularly at observations of different types of supernovae and the need for a classification \\ scheme that can systematically accommodate the diversity of stellar explosions and progenitors.
}

S upernovae $(\mathrm{SNe})$ - the explosive deaths of stars - are some of the oldest recorded astronomical events, and to date almost 63,000 have been catalogued. With such numbers, an observations-based classification system is essential, and the system commonly used today originated in the 1940s when Rudolph Minkowski and Fritz Zwicky split supernovae according to whether hydrogen lines were present in their spectra (type II) or not (type I). (Types III-V were also defined, but they did not stand the test of time.) As the number of observed $\mathrm{SNe}$ grew, further, more subtle, differences became apparent, requiring the introduction of subclasses: type-I SNe became classified as Ia, Ib and Ic and type-II SNe were split into IIP, IIL, IIb and IIn. And still that was not enough to encompass the diversity of supernova spectra and lightcurve evolution. Now there are Ic-bl, Ibn, Ia-CSM, Iax and even .Ia (yes, that is a decimal point in front of the I). There are 'peculiar' SNe. There are $\mathrm{SNe}$ that transcend this entire classification scheme because of their extreme

luminosity - the relatively recent class of superluminous SNe (SLSNe) - or because of their extremely rapid evolution - the fast blue optical transients (FBOTs). There are transient events in the sky that look like supernovae, but are not bright enough: the supernova imposters. What is clear here is that $\mathrm{SNe}$ are as different as you and I, but they also have things in common. And what is eminently clear is that $\mathrm{SNe}$ really need a new taxonomy, because insuperably more $\mathrm{SNe}$ are to be found in current and nextgeneration surveys that will likely reveal that observed supernova properties lie on a continuum, dissolving the boundaries of the conventional classification scheme.

This issue of Nature Astronomy features a Focus on supernovae. The Focus contains three Review Articles on the observed properties of different types of supernova, organized by their astrophysical origin rather than their spectral or photometric appearance. Saurabh Jha, Kate Maguire and Mark Sullivan summarize the latest observational findings in a Review Article on thermonuclear supernovae, particularly those resulting from the detonation of a white dwarf (type Ia). These supernovae have optical lightcurves that are so homogeneous that they have long been used to measure distance, providing the rungs on the cosmic distance ladder in nearby galaxies that enabled the expansion rate of the Universe to be calculated. In a companion Review Article, Cosimo Inserra looks at observations of extreme supernovae, incorporating the SLSNe and FBOTs mentioned above. SLSNe, because of their superior luminosities, may provide the next generation of distance markers. However, whether they can be standardized in the manner of type-Ia SNe still remains to be seen. The third Review Article of the trio, from Maryam Modjaz, Claudia Gutiérrez and Iair Arcavi, takes a slightly different approach. It deals with corecollapse supernovae, resulting from the explosion of massive stars, but looks forward to new approaches in observing them. These three Review Articles, clustered around the cornerstones of physics-based SN classifications, are bolstered by a Comment from Andrea Pastorello and Morgan Fraser on supernova imposters. These not-quitesupernovae are relatively recent additions to the wider transient zoo, which itself is growing year by year.

The supernova landscape is undoubtedly set to change. Currently operational widefield surveys such as Pan-STARRS, CRTS, ATLAS, ASAS-SN and ZTF are producing thousands of SN candidates. Although little has been said about it, the Gaia satellite, too, is detecting thousands of local SNe. The Large Synoptic Survey Telescope (LSST), due to begin operations within the next five years, is expected to find 3-4 million $\mathrm{SNe}$ - that's roughly one thousand per night! Euclid, the European Space Agency's deep sky surveyor that will operate contemporaneously with LSST, has the capability of detecting 100 SLSNe out to redshift $z \approx 3.5$. The ongoing Dark Energy Survey has already reported nearly $500 \mathrm{SNe}$, but expects to find many more, at moderate redshifts.

There will be so many transient detections in our night sky that humans will not be able to process the numbers, and separate out the most promising candidates. ZTF is already producing $\sim 100,000$ transient alerts per night. We will increasingly be relying on machine learning algorithms not only to identify events, but also to classify the events in real - and the least possible - time, in order to rapidly assign more expensive follow-up resources (such as spectroscopy) - getting as close as possible to the ' $b$ ' of the 'bang' is very important for characterizing supernova explosions, for example. Emille Ishida summarizes the work on this front in a Comment in the current issue.

It is not only an increase in numbers that will provide breakthroughs in the studies of $\mathrm{SNe}$, it is also the information that will be available per event. Perhaps the chief aim of SN studies is to identify the progenitors of the different types of SN. Thermonuclear $\mathrm{SNe}$ involve a white dwarf in a binary star system, but the nature of the companion star is not well constrained. Core-collapse (type Ib, Ic and II) and SLSNe most likely both result from the collapse of a massive star, presumably a red supergiant, luminous blue variable or a Wolf-Rayet star, but firm associations between $\mathrm{SNe}$ and their progenitors are rare. Pre-explosion images are key, but they need to be deep to detect any progenitors, and ideally over multiple epochs to study progenitor variability. Currently the number of SNe that meet these criteria can be counted on two hands. Post-explosion time-series monitoring is also needed, since there is growing evidence that SN classifications change with time. LSST in particular should change this situation, with deep ( $r \approx 27.5 \mathrm{mag})$, multiband, approximately half-sky coverage and consistent long-term monitoring at a high cadence. Therefore, we can hope for an imminent future rich in observational diversity, full of new physical understanding, open to surprising discoveries, and with a principled classification scheme that can accommodate all this and more.

Published online: 7 August 2019 https://doi.org/10.1038/s41550-019-0877-X 